\title{
THE COMPREHENSIVE MUSCULAR ACTIVITY PROFILE (CMAP): ITS HIGH SENSITIVITY, SPECIFICITY AND OVERALL CLASSIFICATION RATE FOR DETECTING SUBMAXIMAL EFFORT ON FUNCTIONAL CAPACITY TESTING*
}

\author{
Robert J. Gatchel, Ph.D., ABPP** \\ Mark D. Ricard, Ph.D.*** \\ Dhruti N. Choksi***, Jain Mayank***, and Krista Howard**, Ph.D. Candidate \\ ** Department of Psychology, College of Science, The University of Texas at Arlington \\ *** Department of Kinesiology, The University of Texas at Arlington
}

Address all Correspondence to:

Robert J. Gatchel, Ph.D., ABPP

Professor and Chair, Department of Psychology, College of Science

The University of Texas at Arlington

817-272-2541(V); 817-272-2364(FAX)

gatchel@uta.edu

*This research was supported in part by Grants No. 1K05 MH71892 and 2R01 MH 046452 from the National Institutes of Health, as well as a Research Grant from Medical Technologies, Unlimited. 


\begin{abstract}
Introduction. A traditional problem faced by clinicians attempting to objectively measure musculoskeletal disorders such as low back pain, where there is often primarily soft tissue involvement, is that psychosocial factors (e.g., fear-avoidance, secondary gain) frequently influence the experience/reporting of pain. Nevertheless, there is still a great need for the quantification of physical function, with appropriate criteria in place, in order to help assess both physical impairment and therapeutic endpoint following treatment. One such potentially objective measure is surface electromyographic (sEMG) recordings during purposeful muscular activity and resting states. The present randomized controlled study assessed the potential validity of a new sEMG approach — the Comprehensive Muscular Activity Profile (CMAP)—by addressing the following question: Can the CMAP accurately document whether a subject is exerting appropriate muscular effort during range-of-motion and lifting testing, or is submaximum effort being exerted? Methods. Eighty healthy volunteers were randomly assigned to either: (1) an instruction group encouraging maximum effort on the tests; or (2) an instruction group encouraging "faking" and not putting in maximum effort on the tests. Therapists, who then administered the CMAP protocol (range-of-motion and lifting tests), were kept blind to subject group assignment. They were also asked to complete a rating scale evaluating whether subjects were exerting maximum effort after all the tests were completed. $\underline{\text { Results. }}$. In differentiating between the 2 instruction groups, the CMAP demonstrated high levels of sensitivity [predicting maximum effort on all tests (ranging from 84.6\% to 94.9\%)]. In contrast, the sensitivity of the therapists' ratings was much lower (ranging from only $72.5 \%$ to $80.0 \%$ ). Most importantly, when the CMAP data and therapists' ratings were combined, logistic regression analyses revealed high rates of sensitivity (94.4\%-97.2\%), specificity $(84.6 \%-92.3 \%)$,
\end{abstract}


and overall classification (90.7\%-93.3\%). Conclusion. The results of this study demonstrate the potential utility of the CMAP, combined with therapist ratings, as a valid method of objectively quantifying subject muscular performance and effort during lumbar range-of-motion and lifting tasks.

KEY WORDS: lumbar spine; sEMG; comprehensive muscular activity profile; quantification of function. 
Whenever one evaluates painful spinal disorders, especially in workers' compensation or personal injury populations, the degree of physical impairment must be considered for employment/injury compensation issues. Impairment refers to the alteration of an individual's usual health status, due to anatomic or pathologic abnormalities. In the lumbar spine, it is often assessed by measuring range-of-motion, strength, lifting capacity, aerobic capacity, as well as measures of human performance capability [1]. However, there is currently still no universal agreement about what measures should be used in impairment assessment. The American Medical Association identified only range-of-motion in earlier versions of its Guides to the Evaluation of Permanent Impairment, but it is no longer included in the most recent $6^{\text {th }}$ edition.

A traditional problem faced by clinicians attempting to objectively measure musculoskeletal disorders such as low back pain (LBP), where there is often primarily soft tissue involvement, is that psychosocial factors frequently influence the experience/reporting of pain [2]. Examples of such factors are neuromuscular inhibition due to fear-avoidance of movement, secondary gain, etc. $[3,4]$. Nevertheless, there is still a great need for the quantification of physical function, with appropriate validity criteria in place, in order to help assess both impairment and a therapeutic endpoint following treatment. One such potentially objective measure that has received a great deal of attention over the years has been surface electromyographic (sEMG) recordings during purposeful muscular activity and resting states. There are, though, some differing opinions concerning its utility in differentiating between low back pain patients and normals [5, 6]. Most recently, Geisser, Ranavaya et al. [7] concluded, on the basis of a meta-analysis of the extant scientific literature, that the results were quite mixed, and recommended that "Further research is needed to determine the combination of measures 
that are cost-effective, reliable, valid and discriminate with a high degree of accuracy between healthy persons and those with LBP" (p. 711). Thus, the potential utility of sEMG recordings, which are safe, non-invasive and potentially objective measures of muscle functioning/impairment in the lumbar spine, has not yet been realized. In an attempt to achieve this realization, the Comprehensive Muscular Activity Profile (CMAP) was developed [8]. As an integral part of this attempt, it was decided to develop a firm empirical foundation for its utility and reliability. The first such foundation-building effort will be reported in the present investigation. The basic question addressed was the following: Can the CMAP accurately document whether a subject is exerting appropriate muscular effort during range-of-motion and lifting testing, or is submaximum effort being exerted?

\section{METHODS}

\section{$\underline{\text { Subjects }}$}

Subjects consisted of 80 healthy volunteers (40 males and 40 females) who were recruited through advertisements posted throughout a major University. Subjects were excluded if they had an injury that prohibited normal trunk range-of-motion, or a muscle or joint injury that prohibited them from performing maximal isometric low back strength testing. The 80 subjects were randomly assigned to either : (1) an instruction group encouraging maximum effort on the tests; or (2) an instruction group encouraging "faking" and not putting in maximum effort on the tests. One subject had to be subsequently excluded from the sample because of datatransfer problems, leaving a total sample size of 79. The therapists' ratings for three subjects were not recorded, and therefore were omitted from those particular analyses. Table 1 presents the basic demographic characteristics of these two groups. Chi-square analyses (for dichotamous 
variables) and independent t-tests (for continuous variables) revealed no statistically significant differences between these two groups.

\section{INSERT TABLE 1 ABOUT HERE}

\section{$\underline{\text { Procedure }}$}

After completing the Institution's Research Board's Informed Consent, as well as a Health Status Questionnaire, subjects were randomly assigned to one of the two instructions groups. The therapists [two graduate students (DC and JM) in the Department of Kinesiology, who each saw an equal number of subjects in each group] administered the CMAP protocol, and were kept blind as to group assignments.

Instructions Encouraging Maximum Effort Group. "The major purpose of this study is to evaluate a new measure of muscle activity while you are bending your back. You will be asked to bend forward, backward and sideways while this muscle activity is being recorded on a computer. A series of electrodes will be placed on your skin to measure this muscle activity. This recording is completely safe. This measurement method is going to be used in the future with people who have hurt their backs at work, or while performing some other activity related to work, or due to an accident. However, before it can be used, we need to get a baseline data from people with healthy backs, such as yours, to compare against. Therefore, I want you to bend as much as you can when instructed, so that we have a good measure of maximum bending from someone as healthy as you. Does that make sense? Therefore, please follow the instructions given by the therapist carefully, and bend as much as you can in the direction requested." 
Instructions Encouraging Submaximum Effort ("Faking") Group. "The major purpose

of this study is to evaluate a new measure of muscle activity while you are bending your back. You will be asked to bend forward, backward and sideways while this muscle activity is being recorded on a computer. A series of electrodes will be placed on your skin to measure this muscle activity. This recording is completely safe. This measurement method is going to be used in the future with people who have hurt their backs at work, or while performing some other activity related to work, or due to an accident. Sometimes, people want to "fake" being hurt in order to get out of work. To do this, they will not bend their backs as far as they can so that they will appear to be having problems with their backs. What I want you to do is imagine that you want the therapist to think that you have a bad back and, therefore, do not bend as much as you actually can. In other words, I want you to "fake" on the test and not put in full effort. Your job is to fake it but, also, not make the therapist suspicious that you are faking. Does that make sense? Therefore, please follow the instructions given by the therapist, and bend in the direction requested, but also "hold back a little" so that you do not give maximum effort and you do not make the therapist suspicious."

After the testing protocol was completed, the therapists were required to fill out a brief questionnaire asking: "Did this subject appear to be 'faking' the test to 'look bad on it' at any time during the testing session?" They rated their response on a five-point Likert scale, with the extreme anchors labeled "Definitely No" and "Definitely Yes," and the middle anchor labeled "Could Not Tell."

CMAP Protocol

All subjects were administered three trials of each of the following:

- Rest sitting 
- Rest standing

- Trunk flexion/extension

- Rest standing

- Trunk rotation, right and left

- Rest standing

- Lateral trunk bending, right and left

- Rest standing

- Isometric low back strength testing, underhand grip

- Rest standing

- Isometric low back strength testing, overhand grip

- Rest standing

- Rest sitting

All data were continuously uploaded to the Medical Technologies computerized system where muscle activity scoring/analyses were blindly conducted. All final CMAP data were then transferred to a data spreadsheet for subsequent statistical analyses by an individual (KH) who was "blind" as to the basic experimental hypotheses.

The CMAP Technology has an FDA 510-K Class II approval, as well as approval from Underwriters Laboratory. It is a stand-alone dynamic muscle function monitoring system, with a number of EMG sensors connected to various parts of the subject's body for data collection. Prior to attaching EMG electrodes, the electrode placement sites were shaved, abraded and cleaned with an isopropyl alcohol pad to reduce skin impedance. Surface electrodes were then placed over the belly of the following muscles for the right and left side of the body, with the electrodes aligned in the direction of the muscle fibers: par spinal, quadrates, labarum, gluteus 
maximums, rectus abdominal, abdominal oblique and biceps femora's. The data were then directly fed into a system for acquiring, conditioning and transforming sensor data. Analyzed signals included EMG readings, motion detection and muscle strength measurements. The system acquires continuous analog signals and then digitizes these signals by sampling at a rate of $15 \mathrm{KHz}$. These data are then transferred to a notebook PC for processing using proprietary software. The CMAP identifies valid effort by assessing the morphology and quantifying the EMG signal generated from a muscle or muscle group during the performance of a test. A "compliant" morphology is one in which the characteristic crescendo/decrescendo wave form appears when a muscle approaches its endpoint of range, or contracts against isometric resistance. It should also be noted that the system has dedicated circuitry to filter/shield out background noise (from the power supply, cabling, testing equipment, etc.). The EMG signals are differentially amplified with a gain of 2000 in a bandwidth of 1-2500 Hz. The amplifier has an input noise approaching $12 \mu \mathrm{V}$ RMS, and an effective common mode rejection ratio of about $80 \mathrm{~dB}$. Two notch filters eliminate power line pickup at 60 and $120 \mathrm{~Hz}$. The circuit also detects disconnected leads.

$\underline{\text { Data Analysis }}$

After the processing of the EMG data by CMAP's proprietary software (with subject group assignment being kept blind), the data were then transferred to an independent data spreadsheet from which the statistical analyses were conducted. A series of $2 \mathrm{X} 2$ chi-square analyses were conducted (with the rows representing CMAP's determination of valid effort; and the columns representing instruction-group assignment) for range-of-motion, lifting (with underhand grip) and lifting (with overhand grip) It should be noted that the CMAP decision algorithm used in determining valid effort was based on a prior data set, and not on the data 
collected in the present study. For therapists' ratings of perceived effort, two analyses were evaluated. First, a 3 X 2 chi-square analysis was conducted (with the rows representing the therapists' evaluation of effort—with Likert Scale points 1 and 2 representing definitely faking; 3 representing could not tell; and 4 and 5 representing definitely not faking; and the columns representing CMAP's determination of valid effort). Secondly, a 3 X 2 chi-square analysis was conducted (with the rows representing the therapist's evaluation of effort and the columns representing instruction-group assignment). Finally, a series of sequential logistic regression analyses were conducted for each of the three measures to predict to which instruction group the subjects were assigned. The therapists' ratings (using the full 1-5 scale) were entered into the model first, followed by the second block containing the CMAP data. This was done to evaluate if the CMAP data predicted group assignment "over and above" that of the therapists' ratings by evaluating the chi-square value for the second block.

\section{RESULTS}

\section{$\underline{\text { Range-of-Motion (ROM) }}$}

Table 2 presents the chi-square analyses of the CMAP ROM results. As can be seen, there was a significant effect for correct CMAP determination of valid effort, $\mathrm{X}^{2}=25.96, \mathrm{p}<.001$, with a high degree of sensitivity $(94.9 \%)$. In contrast, even though there was also a significant effect for the therapists' ratings, $\mathrm{X}^{2}=31.59, \mathrm{p}<.001$ (Table 3 ), the sensitivity was much lower $(72.5 \%)$.

\section{INSERT TABLES 2 AND 3 ABOUT HERE}

\section{Lifting-Underhand Grip}


For the lifting measure (Table 4), there was again a significant effect for correct CMAP determination, $\mathrm{X}^{2}=32.14, \mathrm{p}<.001$ (sensitivity of $84.6 \%$ ), as well as for therapists' ratings (Table $5 ; X^{2}=22.33, p<.001$; sensitivity of $\left.79.5 \%\right)$.

\section{INSERT TABLES 4 AND 5 ABOUT HERE}

\section{Lifting-Overhand Grip}

Tables 6 and 7 present these lifting-measure results for the CMAP and therapists' ratings, respectively. Again, there were significant results for both, with slightly higher sensitivity associated with the CMAP.

\section{INSERT TABLES 6 AND 7 ABOUT HERE}

\section{Therapists' Ratings and Group Assignment}

Table 8 represents the therapists' overall ratings compared directly to the group assignment (whether the subject was told to give maximum or submaximum effort). As revealed by the analysis, the ratings by the therapists to accurately predict group placement was statistically significant. The ability of the therapists to predict maximum effort was greater than the ability to predict submaximum effort, as shown by the sensitivity (97.3\%) and specificity $(62.5 \%)$.

\section{INSERT TABLE 8 ABOUT HERE}




\section{Combined CMAP and Therapists' Ratings}

Table 9 presents the sequential regression models for each of the three measures. For each model, the therapists' ratings were entered into Block 1, and the CMAP data were entered into Block 2. As can be seen by the chi-square values for Block 2 for each of the three measures, the addition of the CMAP data to the model was significant "over and above" that of the therapists' ratings alone.

In order to determine whether sensitivity, specificity and overall classification rate could be increased, the final blocks of each of the sequential logistic regression models were evaluated. As can be seen in Table 10, this resulted in a substantial increase in all three indices, across all three measures for these combined data (highlighted in bold). It is quite rare to have such high values for sensitivity, specificity and overall classification rate for all three measures.

\section{INSERT TABLES 9 AND 10 ABOUT HERE}

\section{DISCUSSION}

The results of the present randomized controlled study demonstrated that the CMAP system is a potentially useful method for evaluating lumbar range-of-motion and lifting capacity, while also documenting subject effort during these performance tasks. As noted earlier, past research on the use of sEMG measures has been quite mixed in terms of their validity to discriminate, with a high degree of accuracy, between healthy persons and those with LBP [7]. One major reason for this has been the fact that psychosocial factors (e.g., fear-avoidance of movement, secondary gain, etc.) often influence the experience/reporting of pain. It is therefore essential to have a method to gauge whether subjects are exerting maximum effort during 
purposeful muscle activity. Data from the current investigation reveal the ability to do just that.

Of course, future research will need to further address this important issue by evaluating a patient group with LBP. This clinical research is currently being planned.

With the growing annual costs associated with the diagnosis and care of musculoskeletal disorders amounting to tens of billions of dollars in the United States alone [e.g., [2]], there is a vital need to develop objective and valid measures to quantify physical function in patients with these disorders. This will help assess both physical impairment (needed for addressing compensation issues), as well as use in determining a therapeutic endpoint following treatment. Moreover, the biopsychosocial nature of these chronic disorders also makes it essential to "factor in" the potential contribution of psychosocial issues in such an evaluation process [9]. Again, the CMAP provides such an objective and appealing approach to a biopsychosocial evaluation. It can also be a cost- and time-effective alternative to other methods.

Indeed, there have been numerous other physical functioning measurement devices and protocols developed over the years for range-of-motion and lifting capacity. However, in a review of quantification of function, Flores, Gatchel and Polatin [1] concluded that these measures of “...human performance are helpful but not infallible in guiding impairment evaluation, largely because of limited ability to assess effort in many of the tests" (p. 1626). One of the most critical and difficult aspects of these measurements is the subject's effort, which has a profound effect on performance. Similarly, Hazard, Reeves and Fenwick [10], in their evaluation of subjects' effort on isometric, isokinetic and isoinertial tests of lifting capacity, found that indices of maximum/submaximum effort in these lifting protocols are quite limited and variable. As demonstrated in the present study, the CMAP may overcome such limitations. 
Finally, it should also be pointed out that the combined CMAP and therapists' ratings of maximum/submaximum performance yielded the highest levels of sensitivity, specificity and overall classification rates for all three measures evaluated in this project (all in the .90 range). It is also quite important to note that, no matter how valid a physiological measurement tool is, there is always the important "device-to-subject interface" that can affect the accuracy of the measurement process [11]. Therefore, it will be of utmost importance to have experienced examiners who have a keen appreciation for the importance of the extreme care needed in skin preparation, precise electrode placement, and proper electrical connection when administering the tests. Also, therapists' experience allows them to better independently evaluate patient effort during the testing. This awaits future research.

Of course, in any clinical research study, there are often certain limitations that can be noted. In the present investigation, one such limitation was the fact that all study participants were healthy college students who were unlikely to have experienced low back pain. Also, the therapists may not have expected that these participants would show any legitimate problem with physical functioning; thus, sub-maximal effort might have been more easily detected in this population. Relatedly, in future research, it will be of great interest to evaluate an older population in which psychosocial factors may have a more prominent and direct influence on the physiological and functional components of the pain experience.

In conclusion, the results of this investigation clearly demonstrate the utility of the CMAP, combined with the ratings of experienced therapists, as a method of objectively quantifying muscular performance and effort during lumbar range-of-motion and lifting tasks. Because of the tremendous socioeconomic impact of musculoskeletal disabilities such as low back pain, developing a valid evaluation tool to assess both impairment and a therapeutic 
endpoint following treatment is essential. The CMAP displays great promise for the area of musculoskeletal disability and impairment evaluation. 


\section{REFERENCES}

1. Flores L, Gatchel RJ, Polatin PB. Objectification of functional improvement after nonoperative care. Spine. 1997;22(14):1622-33.

2. Mayer TG, Gatchel RJ, Polatin PB, editors. Occupational musculoskeletal disorders: Function, outcomes and evidence. Philadelphia: Lippincott Williams \& Wilkins; 2000.

3. Leeman G, Polatin P, Gatchel R, Kishino N. Managing secondary gain in patients with pain-associated disability: A clinical perspective. Journal of Workers Compensation. 2000;9:2544.

4. Rainville J, Sobel J, Hartigan C, Wright A. The effect of compensation involvement of the reporting of pain and disability by patients referred for rehabilitation of chronic low back pain. Spine. 1997;22(17):2016-24.

5. Haig AJ, Gelblum JB, Rechtine JJ, Gitter AJ. Technology assessment: The use of surface emg in the diagnosis and treatment of nerve and muscle disorders. Muscle \& Nerve. 1996;19:392-5.

6. Pullman SL, Goodin DS, Marquinez AI, Tabbal, S., Rubin M. Clinical utility of surface emg: Report of the therapeutics and technology assessment subcommittee of the american academy of neurology. Neurology. 2000;55:171-7.

7. Geisser ME, Ranavaya M, Haig AJ, Roth RS, Zucker R, Ambroz C, Caruso M. A metaanalytic review of surface electromyography among persons with low back pain and normal, healthy controls. Journal of Pain. 2005;6:711-26.

8. Medical Technologies Unlimited. Comprehensive muscular activity profile. 2008 [cited 2008 8-12-08]; Available from: http://www.med-tek.com 
9. Gatchel RJ. Musculoskeletal disorders: Primary and secondary interventions. J Electromyogr Kinesiol. 2004;14:161-70.

10. Hazard RG, Reeves V, Fenwick JW. Lifting capacity: Indices of subject effort. Spine. 1992;17:1065-70.

11. Mayer TG, Kondraske G, Beals SB, Gatchel RJ. Spinal range of motion. Accuracy and sources of error with inclinometric measurement. Spine. 1997;22(17):1976-84. 
Table 1 Basic Demographic Data of the Subject Cohort

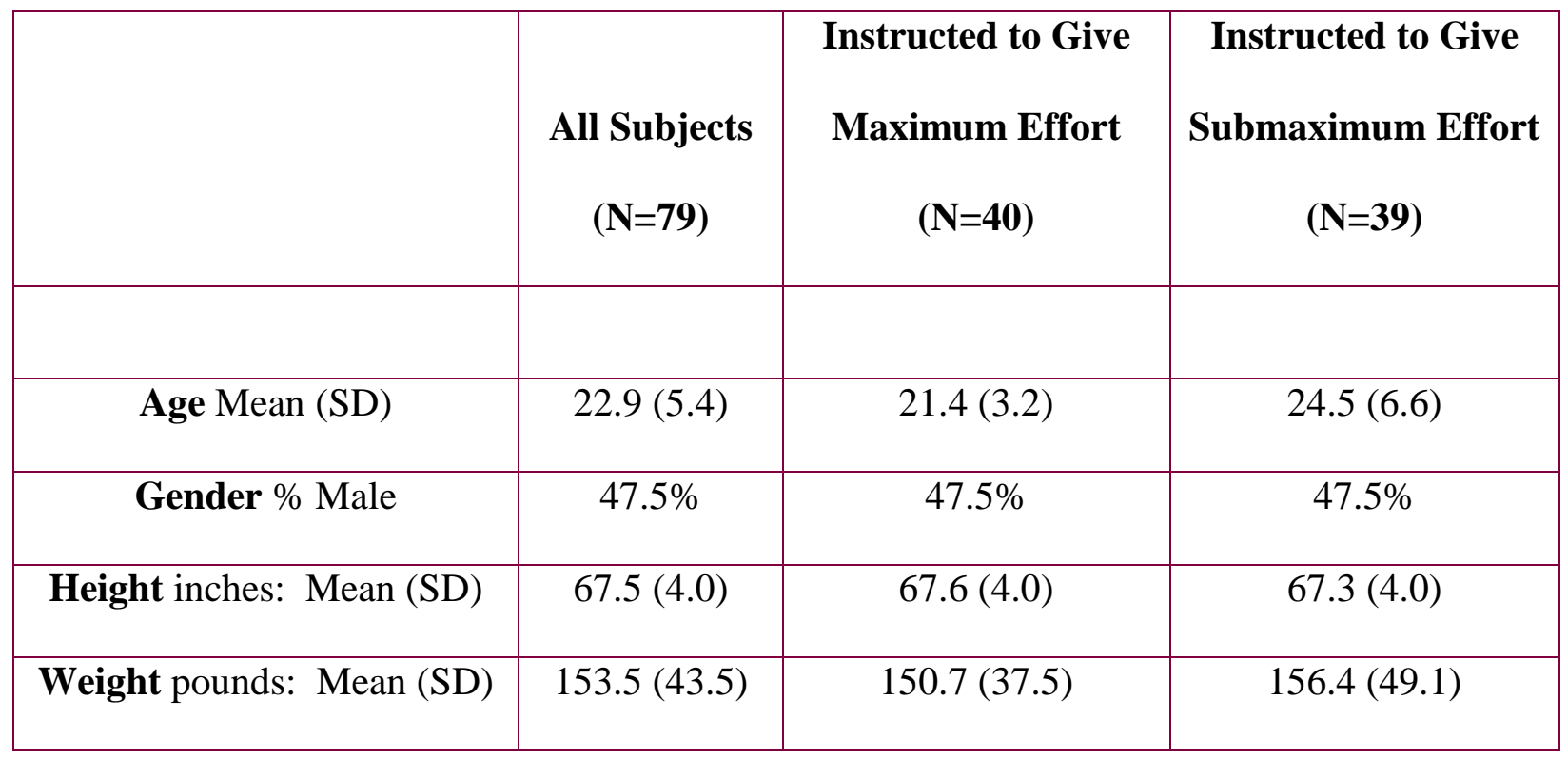


Table 2. Quantified ROM Results-CMAP

\begin{tabular}{|l|c|c|}
\hline & $\begin{array}{c}\text { Maximum Effort } \\
\text { Instructions }\end{array}$ & $\begin{array}{c}\text { Submaximum Effort } \\
\text { Instructions }\end{array}$ \\
\hline & 37 & 16 \\
\hline CMAP: Maximum Effort & 2 & 23 \\
\hline CMAP: Submaximum Effort & & \\
\hline
\end{tabular}

$\mathrm{X}^{2}=25.96, \mathrm{p}<.001$

Sensitivity (Predicting Maximum Effort) $=94.9 \%$

Specificity (Predicting Submaximum Effort $)=59.0 \%$

Overall Classification $=76.9 \%$ 
Table 3. Quantified ROM Results-Therapists Ratings

\begin{tabular}{|l|c|c|}
\hline & CMAP-Documented & CMAP-Documented \\
& Maximum Effort & Submaximum Effort \\
\hline Therapist: Definitely Maximum Effort & 37 & 3 \\
\hline Therapist: Could Not Determine & 7 & 2 \\
\hline Therapist: Definitely Submaximum Effort & 7 & 19 \\
\hline
\end{tabular}

$X^{2}=31.59, \underline{p}<.001$

Sensitivity $=72.5 \%$

Specificity $=79.2 \%$

Overall Classification $=74.7 \%$ 
Table 4. Lifting, Underhand Grip-CMAP

\begin{tabular}{|l|c|c|}
\hline & $\begin{array}{c}\text { Maximum Effort } \\
\text { Instructions }\end{array}$ & $\begin{array}{c}\text { Submaximum Effort } \\
\text { Instructions }\end{array}$ \\
\hline & 33 & 8 \\
\hline CMAP: Maximum Effort & 6 & 31 \\
\hline CMAP: Submaximum Effort & & \\
\hline
\end{tabular}

$\mathrm{X}^{2}=32.14, \mathrm{p}<.001$

Sensitivity $=84.6 \%$

Specificity $=79.5 \%$

Overall Classification $=82.1 \%$ 
Table 5. Lifting, Underhand Grip-Therapists' Ratings

\begin{tabular}{|l|c|c|}
\hline & $\begin{array}{c}\text { CMAP-Documented } \\
\text { Maximum Effort }\end{array}$ & $\begin{array}{c}\text { CMAP-Documented } \\
\text { Submaximum Effort }\end{array}$ \\
\hline Therapist: Definitely Maximum Effort & 31 & 9 \\
\hline Therapist: Could Not Determine & 2 & 7 \\
\hline Therapist: Definitely Submaximum Effort & 6 & 20 \\
\hline
\end{tabular}

$X^{2}=22.33, p<.001$

Sensitivity $=79.5 \%$

Specificity $=55.6 \%$

Overall Classification $=68.0 \%$ 
Table 6. Lifting, Overhand Grip-CMAP

\begin{tabular}{|l|c|c|}
\hline & Maximum Effort & Submaximum Effort \\
& Instructions & Instructions \\
\hline CMAP: Maximum Effort & 35 & 8 \\
\hline CMAP: Submaximum Effort & 4 & 31 \\
\hline
\end{tabular}

$X^{2}=37.78, \underline{p}<.001$

Sensitivity $=89.7 \%$

Specificity $=79.5 \%$

Overall Classification $=84.6 \%$ 
Table 7. Lifting, Overhand Grip-Therapists' Ratings

\begin{tabular}{|l|c|c|}
\hline & $\begin{array}{c}\text { CMAP-Documented } \\
\text { Maximum Effort }\end{array}$ & $\begin{array}{c}\text { CMAP-Documented } \\
\text { Submaximum Effort }\end{array}$ \\
\hline Therapist: Definitely Maximum Effort & 32 & 8 \\
\hline Therapist: Could Not Determine & 3 & 6 \\
\hline Therapist: Definitely Submaximum Effort & 5 & 21 \\
\hline
\end{tabular}

$X^{2}=25.02, \underline{p}<.001$

Sensitivity $=80.0 \%$

Specificity $=60.0 \%$

Overall Classification $=70.7 \%$ 
Table 8. Therapists' Ratings and Group Assignment

\begin{tabular}{|l|c|c|}
\hline & Maximum Effort & Submaximum Effort \\
& Instructions & Instructions \\
\hline Therapist: Definitely Maximum Effort & 36 & 5 \\
\hline Therapist: Could Not Determine & 0 & 10 \\
\hline Therapist: Definitely Submaximum Effort & 1 & 25 \\
\hline
\end{tabular}

$X^{2}=55.56, \underline{p}<.001$

Sensitivity $=97.3 \%$

Specificity $=62.5 \%$

Overall Classification $=79.2 \%$ 
Table 9. Sequential Logistic Regression for the Three Measures

\begin{tabular}{|c|c|c|c|c|c|}
\hline $\begin{array}{l}\text { Variable - } \\
\text { Quantified ROM }\end{array}$ & B & Wald & $p$ value & $\begin{array}{l}\text { Odds } \\
\text { Ratio }\end{array}$ & $95 \%$ CI \\
\hline Block 1: Therapists' Ratings & 1.610 & 14.749 & .000 & 5.004 & $2.2,11.4$ \\
\hline Block 2: CMAP Data & 2.635 & 4.021 & .045 & 13.945 & $1.1,183.2$ \\
\hline Constant & -3.849 & 20.132 & .000 & & \\
\hline \multicolumn{6}{|c|}{ ROM Block 2: $\mathrm{X}^{2}=4.761, \mathrm{p}=.029$} \\
\hline $\begin{array}{l}\text { Variable - } \\
\text { Underhand Lift }\end{array}$ & B & Wald & $p$ value & $\begin{array}{l}\text { Odds } \\
\text { Ratio }\end{array}$ & $95 \% \mathrm{CI}$ \\
\hline Block 1: Therapists' Ratings & 1.623 & 14.186 & .000 & 5.070 & $2.2,11.8$ \\
\hline Block 2: CMAP Data & 3.058 & 9.486 & .002 & 21.293 & $3.0,149.1$ \\
\hline Constant & -4.900 & 18.008 & .000 & & \\
\hline \multicolumn{6}{|c|}{ Underhand Lift Block 2: $X^{2}=12.061, p=.001$} \\
\hline $\begin{array}{l}\text { Variable - } \\
\text { Overhand Lift }\end{array}$ & B & Wald & $p$ value & $\begin{array}{l}\text { Odds } \\
\text { Ratio }\end{array}$ & $95 \% \mathrm{CI}$ \\
\hline Block 1: Therapists' Ratings & 1.617 & 12.666 & .000 & 5.038 & $2.1,12.3$ \\
\hline Block 2: CMAP Data & 3.111 & 9.956 & .002 & 22.449 & $3.3,155.1$ \\
\hline Constant & -4.757 & 17.937 & .000 & & \\
\hline \multicolumn{6}{|c|}{ Overhand Block 2: $X^{2}=12.312, \underline{p}<.001$} \\
\hline
\end{tabular}


Table 10. Sensitivity, Specificity and Overall Classification Values for the Combined CMAP and Therapists

\begin{tabular}{|c|c|c|c|}
\hline \multicolumn{4}{|c|}{ Combined Ratings (Bold) for Each of the Three Measures } \\
\hline & Sensitivity & Specificity & Overall Classification \\
\hline CMAP & $94.9 \%$ & $59.0 \%$ & $76.9 \%$ \\
\hline Therapist & $72.5 \%$ & $79.2 \%$ & $74.7 \%$ \\
\hline Combined* & $\mathbf{9 4 . 4 \%}$ & 92.3\% & 93.3\% \\
\hline \multicolumn{4}{|c|}{$\underline{\text { Lifting - Underhand }}$} \\
\hline CMAP & $84.6 \%$ & $79.5 \%$ & $82.1 \%$ \\
\hline Therapist & $79.5 \%$ & $55.6 \%$ & $68.0 \%$ \\
\hline Combined* & $97.2 \%$ & $84.6 \%$ & $90.7 \%$ \\
\hline \multicolumn{4}{|c|}{ Lifting - Overhand } \\
\hline CMAP & $89.7 \%$ & $79.5 \%$ & $84.6 \%$ \\
\hline Therapist & $80.0 \%$ & $60.0 \%$ & $70.7 \%$ \\
\hline Combined* & $97.2 \%$ & $89.7 \%$ & 93.3\% \\
\hline
\end{tabular}

